\title{
Sensing the Environmental Neighborhoods
}

\section{Mobile Urban Sensing Technologies (MUST) for High Spatial Resolution Urban Environmental Mapping}

\author{
Maider Llaguno-Munitxa ${ }^{1,2(凶)}$ and Elie Bou-Zeid ${ }^{2}$ \\ 1 Northeastern University, 11 Leon St, Boston, MA 02115, USA \\ m.1lagunomunitxa@northeastern.edu, mllaguno@princeton.edu \\ 2 Princeton University, 59 Olden St, Princeton 08540, USA
}

\begin{abstract}
Given the benefits of fine mapping of large urban areas affordably, mobile environmental sensing technologies are becoming increasingly popular to complement the traditional stationary weather and air quality sensing stations. However the reliability and accuracy of low-cost mobile urban technologies is often questioned. This paper presents the design of a fast-response, autonomous and affordable Mobile Urban Sensing Technology (MUST) for the acquisition of high spatial resolution environmental data. Only when accurate neighborhood scale environmental data is affordable and accessible for architects, urban planners and policy makers, can design strategies to enhance urban health be effectively implemented. The results of an experimental air quality sensing campaign developed within Princeton University Campus is presented.
\end{abstract}

Keywords: Urban sensing · Environmental neighbourhood · Urban microclimate $\cdot$ Air quality

\section{Introduction}

Recent reports have demonstrated that intraurban and neighbourhood scale urban environmental gradients can be greater than between district or city-rural differences [1, 2], and these gradients have been associated with adverse health outcomes, including cardiovascular and respiratory disease, mortality and the exacerbation of asthma and chronic obstructive pulmonary disease [3-5]. In this context, the acquisition of urban environmental data at high spatial resolutions is critical to accurately characterize these urban environmental gradients at the neighbourhood scale and identify the areas of the city with most compromised urban environmental conditions. In the last decade, many municipalities have invested in extensive low-cost urban environmental sensing networks. That is the case with the $\mathrm{C} 40$ Cities air quality network led by the cities of London in the UK and Bengaluru in India [6], or the Array of Things (AoT) project in Chicago [7], amongst others. The quality control protocols required to guarantee an acceptable performance of low-cost sensing networks, however, make their maintenance challenging and costly. And most importantly, stationary sensors have proven incapable of identifying the spatial gradients present at the neighbourhood scale $[1,2,8]$. 
An increasingly popular alternative to low cost fixed environmental sensing networks, are mobile urban environmental sensing technologies. Mobile urban sensing is not a new sensing protocol [9-11]; however, in the last decade it has become increasingly popular $[12,13]$ given the limited investment required to cover a large urban area at a high spatial resolution. Recent mobile sensing experiments include campaigns developed in Berkeley, Houston and London [14-16] amongst others. As reported by Apte et al. [1], air quality concentrations can be 8 times larger within a street, and with a limited number of vehicle fleets a large urban area $\sim 10 \mathrm{~km}^{2}$ can be effectively mapped in a limited time frame. However, while mobile sensing technologies can potentially unveil the strong spatial gradients present at the neighbourhood scale, standard design criteria for low-cost mobile sensing technologies to accurately characterize these environmental gradients are yet to be defined.

This paper presents an autonomous, affordable, and fast-response mobile urban sensing technology designed to aid our research on the environmental neighbourhoods, defined as the surrounding urban area influencing the environmental quality of a given point in the city [2]. A prior generation of MUST sensing kits was deployed over the Seoul inter-city bus network as part of the 2017 Seoul Architecture and Urbanism Biennale $[17,18]$. The current generation of MUST sensing kits, however, has evolved to focus on the acquisition of fine spatial environmental gradients to reveal the intimate relationship between local urban features and the surrounding environmental quality. On the one hand, the design criteria of the MUST sensing kit has focused on the definition of a mechanical design that guarantees resilience over adverse weather conditions such as overheating and heavy rainfall, while providing sufficient air exchanges to enable fast-response data acquisition. On the other hand, affordability and autonomy have also been two important criteria. Fast-response and low-cost readily available sensors have been used, and magnetic legs and solar panels have been incorporated into the design to guarantee power autonomy and an easy deployment over private vehicles and public transportation networks. The results of an experimental air quality sensing campaign developed within Princeton University Campus to test and demonstrate the capabilities of this technology are presented. MUST sensing kits were deployed over Princeton University printing and mailing service vehicles for mobile road air quality mapping. As displayed in Fig. 1, the collected mobile sensing datasets were stored in real-time over a cloud service and made available through web and mobile applications to wider audiences within campus.

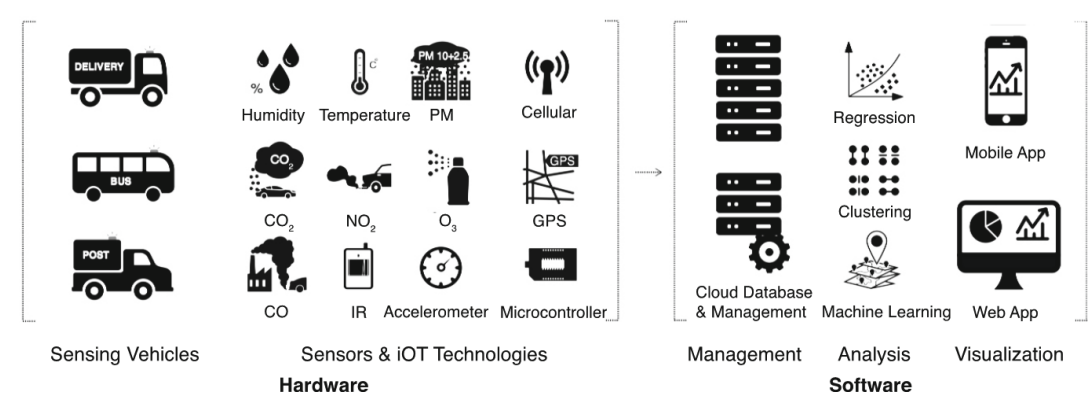

Fig. 1. Conceptual MUST workflow diagram. 


\subsection{Sensing Kit Design}

The MUST sensing kit is an autonomous, affordable and fast-response sensing kit designed to enable low-cost, neighbourhood-scale environmental monitoring campaigns. The sensing kit relies on a solar panel to sustain the power consumption requirements of the system, and thus it can be placed on any vehicle without additional power supplies. To guarantee an easy deployment, strong rare-earth magnet legs have been introduced. Fast-response, readily-available sensors have been used to minimize the investment, and a focus on the design of the enclosure has been made to maximize the responsiveness of the sensing kit to capture the neighbourhood scale environmental gradients, while providing sufficient protection during adverse weather conditions (see Figs. 2 and 3).
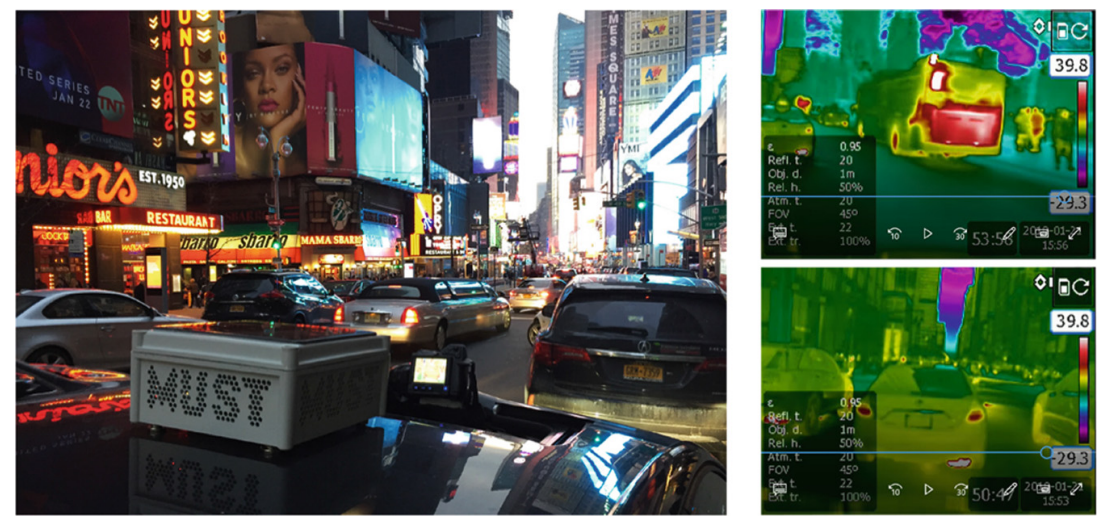

Fig. 2. Deployment of MUST in NYC vehicle. Infrared (IR) video captured in NYC.
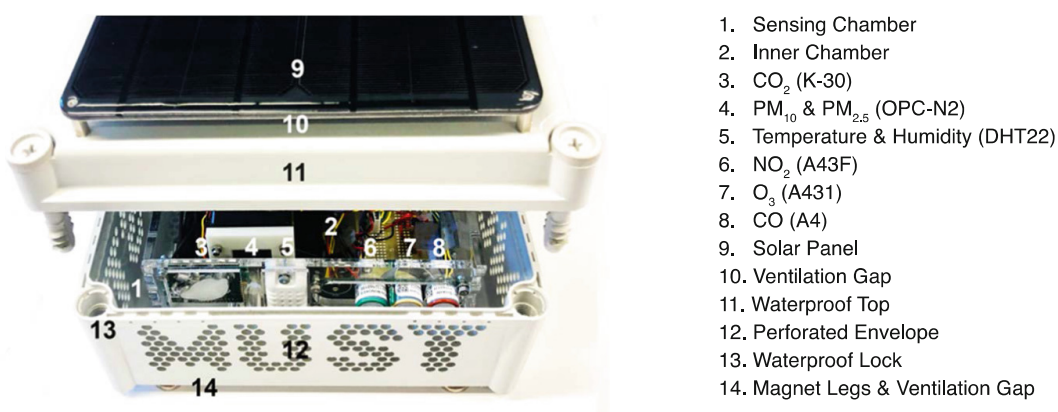

Fig. 3. MUST sensing kit configuration.

\subsubsection{Hardware Design - Enclosure}

The MUST sensing kit container has been designed aiming to protect the interior electronics from water, while enabling sufficient air exchange rate for the effective functioning of the sensors. Built in polyurethane, the exterior box of the MUST sensing kit has 
been perforated on $\approx 30 \%$ of its surface, with openings diameters $\phi \sim 0.5$ " to enable air flow to enter the sensing chamber while stopping the entry of large water droplets. An inner polyurethane shield faces the openings in the outer skin creating an inner chamber to collect the water droplets that enter the box and protect the microcontroller and electric boards, placed in the chamber, from the water. The sensing heads have been located such that their sensing surface protrude into the inner chamber, through the polyurethane shield and facing the side of the box toward the front of the vehicle, facing the incoming flow. A solar panel has been placed on the top of the box occupying the whole surface of the sensing kit to maximize its power collection capacity and guarantee that the sensing kit can operate autonomously.

Seeking a qualitative visualization to understand the ventilation potential of the box and the air exchanges that take place in the sensing chamber located between the perforated outer skin and the inner chamber, fog visualization wind tunnel experiments have been performed in the closed loop wind tunnel located in Princeton University's Instructional Fluid Dynamics Laboratory (see Fig. 4). The tunnel is a modified version of a wind tunnel model 407-A manufactured by Engineering Laboratory Design, Inc (for further details on the tunnel characteristics see [19]). The MUST kit sensing wall was positioned perpendicular to the inlet of the wind tunnel and the tunnel was set to very low wind speed velocities $<1 \mathrm{~m} \mathrm{~s}^{-1}$ to minimize the presence of turbulence in the visualization and mimic slow vehicle movement where air exchange is weakest. Two setups were tested. In the first setup, the fog source was positioned upstream of the MUST sensing kit and was released through a sequence of $\phi \approx 0.25$ " diameter nozzles pointing in the direction of the incoming flow. The nozzles are connected to the fog generator, which keeps the pipe full of fog and is released by the suction force induced by the incoming air flow. For the second setup, the fog was released through a small tube of $\phi \sim 0.35$ " located in the bottom of sensing chamber area. In this case a single release nozzle was used to fill the chamber space with smoke given that the goal was to visualize the efficiency of the sensing area to be cleared of smoke. The top sequence of images included in Fig. 4 shows that once the wind tunnel speed is setup to $\approx 1 \mathrm{~m} \mathrm{~s}^{-1}$, in $<4 \mathrm{~s}$, the sensing chamber is clear of fog. The second row shows the nozzle configuration located in front of the box. The fog generator is started and once the sensing chamber is filled, the wind tunnel is set to $\sim 1 \mathrm{~m} \mathrm{~s}^{-1}$. As with the first configuration, in $<4 \mathrm{~s}$, the chamber is free of fog. Given the low speeds tested in the tunnel, with velocities more representative of a stationary condition than a mobile kit, this study guarantees that when the vehicle is on the move, the air exchange rates will be sufficient for data collection at a rate of $\approx 2 \mathrm{~s}$ (frequency of $\approx 0.5 \mathrm{~Hz}$ ). (see Table 1). Given that the movement of cars will take place in an average intraurban velocity of about $20 \mathrm{~km} \mathrm{~h}^{-1}$ or $5 \mathrm{~m} \mathrm{~s}^{-1}$, the spatial resolution of the measurement will be on the order of $10 \mathrm{~m}$. Therefore, the observed air exchange rate has been considered sufficient.

To evaluate the effect a prolonged exposure to direct sunlight can have on sensor stability and temperature and humidity measurements, the offset in temperature within and outside of the sensing kit was measured in a mobile and stationary vehicle during a summer day within Princeton University campus. In the case of the mobile vehicle, a maximum Temperature $\Delta \approx 1{ }^{\circ} \mathrm{C}$ was observed. In order to enhance heat transfer and avoid overheating, following the studies ventilation gaps were introduced between the 

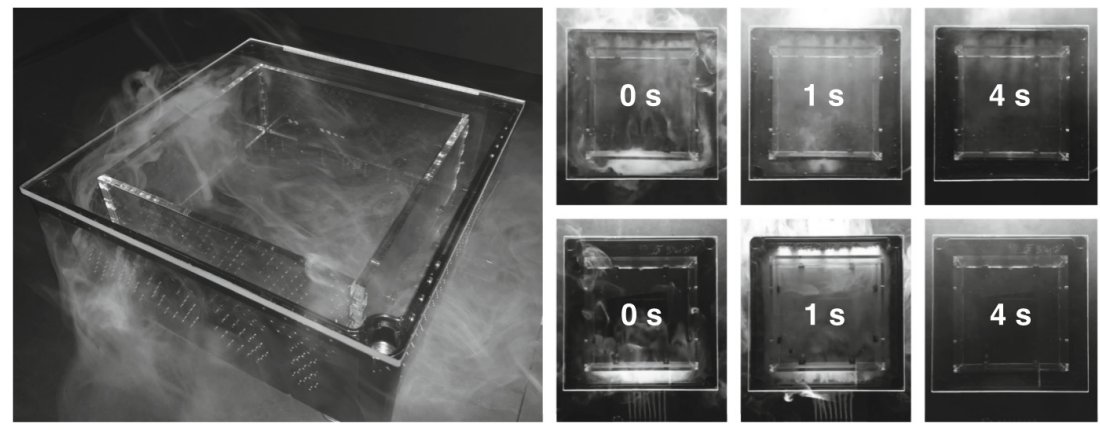

Fig. 4. MUST fog visualization. The image to the left shows the WT setup. The top right sequence shows the setup when the fog emitter is located in the inner sensing chamber. The bottom row shows the setup when the fog emitter nozzles are located in front of the box.

Table 1. MUST sensing kit sensor list, sampling intervals and calibration instruments.

\begin{tabular}{l|l|l|l}
\hline & Commercial Brand & Sampling frequency & Calibration reference sensor \\
\hline Temperature \& Humidity & DHT 22 & $0.5 \mathrm{~Hz}$ & Rotronic MP101A \& Vaisala HMS110 \\
\hline $\mathrm{CO}_{2}$ & CO2Meter K-30 & $0.5 \mathrm{~Hz}$ & LI-COR7500 \\
\hline $\mathrm{PM}_{10, P M_{2.5}}$ & Alphasense OPC-N2 & $0.1 \mathrm{~Hz}$ & Thermo Beta 5014i \\
\hline $\mathrm{NO}_{2}$ & Alphasense NO2-A43F & $0.5 \mathrm{~Hz}$ & Teledyne T500U \\
\hline $\mathrm{O} 3$ & Alphasense OX-A431 & $0.5 \mathrm{~Hz}$ & Teledyne T400 \\
\hline $\mathrm{CO}$ & Alphasense CO-A4 & $0.5 \mathrm{~Hz}$ & Under development \\
\hline
\end{tabular}

vehicle and the bottom of the sensing kit, and between the solar panel and the cap of the sensing kit (see Fig. 3).

\subsubsection{Hardware Design - Sensors}

The sensing kit contains sensors for carbon monoxide, ozone, particulate matter $\left(\mathrm{PM}_{10}\right.$, $\mathrm{PM}_{2.5}, \mathrm{PM}_{1}$ ) nitrogen dioxide, carbon dioxide, temperature and humidity. As described in Table 1, low-cost and readily available sensors that provide a reasonable response time to develop neighborhod scale environmental sensing were chosen. The sensing kits are also equipped with a GPS shield that enables the kits to be geo-localized in real-time. Furthermore, the kit also comprises a cellular antenna that enables the acquired data to be transmitted in near real-time to an online database.

The sensor calibration has been performed following 3 methodologies. The MUST sensing kits were calibrated against i) a reference EPA air quality station located at the Rutgers University campus, ii) in the laboratory at the Princeton University Environmental Fluid Mechanics Lab, iii) and during the measuring campaign through cross-calibration between different MUST sensing kits (see Table 1 for instrument details). 
Figure 5 shows the comparison between the MUST measurements of $\mathrm{CO}_{2}$ and temperature against the reference sensors. The $\mathrm{CO}_{2}$ calibration consistently showed an offset for the different K-30 sensors. The relative humidity readings have been converted to specific humidity for comparability purposes. It is also noted that for the specific humidity, there is an offset between the MUST sensors and the Vaisala HMS110. Based on these comparison, linear recalibrations of the MUST sensors are incorporated into the code to improve data quality. The recalibration for temperature on the other hand required a second-order fit given the distinct trends observed in the data. Finally, in order to enable sensor cross-calibration, for every measuring trip, two MUST sensing boxes were placed next each other. Additionally, an infrared camera was coupled to the MUST sensing boxes to evaluate the impact of the presence of neighboring vehicular exhaust or greenery, or exposure to direct sunlight in the data peaks and valleys.
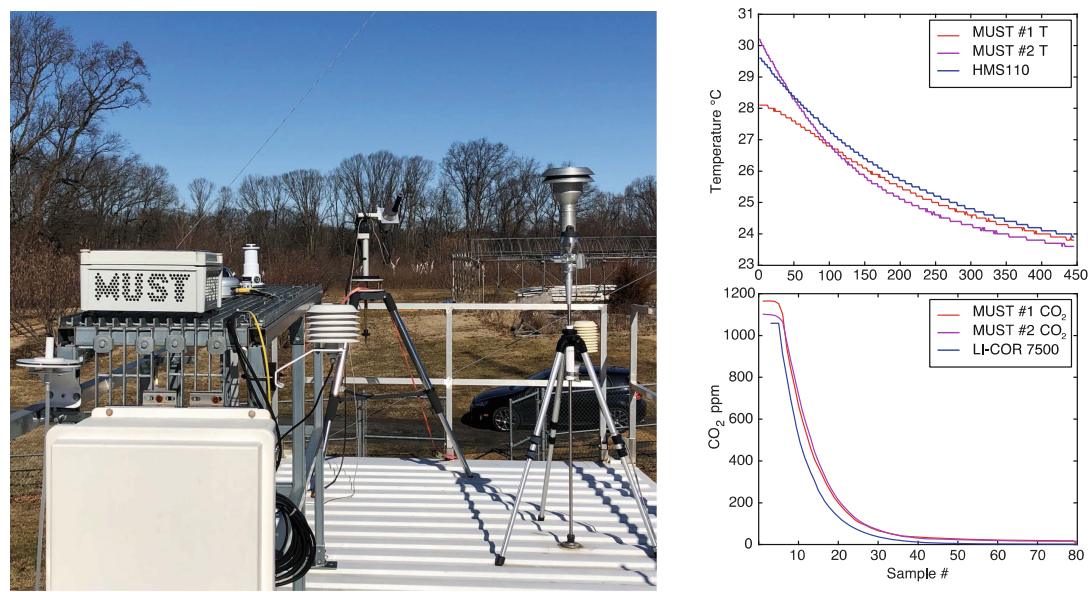

Fig. 5. To the left, the calibration setup against the Rutgers New Brunswick air quality station. To the right, the calibration results in the lab for temperature, and $\mathrm{CO}_{2}$ are shown.

One of the main challenges of the system was to optimize the power consumption to minimize operation maintenance. The code includes a hardware sleep routine which disconnects the cellular connectivity to minimize battery consumption when the battery level reaches a given threshold, and the highest consuming sensors are disconnected through a power relay to minimize the consumption of the kit when the system is sent to sleep. The sleep routine can also be remotely activated if the weather conditions are not desirable, or the vehicle is stationary. A multicell rechargeable lithium battery of 20.000 $\mathrm{mAh}$, guarantees that the system can remain awake for 3 days without being recharged by the solar panel, that is with no daylight.

\subsection{Case Study}

MUST sensing kits were deployed over Princeton university mailing and printing service logistic vans during summer 2019 for the duration of a month. In every measuring day, 
a daily average of $\sim 3-4 \mathrm{~h}$ data was collected. In every occasion, a similar route was followed at least twice, and the collection time was performed from $3 \mathrm{pm}$ onwards to guarantee that the rush hour peaks were also captured. As mentioned in Sect. 1.1.2, two MUST sensing kits where located on the same vehicle for cross-calibration purposes. The data collection was performed at $0.5 \mathrm{~Hz}$ frequency for all sensors, but the PM data were collected at $0.1 \mathrm{~Hz}$. The data were sent to an online and publicly accessible server in real time. When the cellular connectivity of GPS fix was lost, the data were not sent to the server. However, such instances never exceeded $3 \%$ of the collected data volume and thus did not amount to an important loss of information.

In Fig. 6 the air quality data collected during a day on July 2019 within Princeton University central and Forrestal campus are shown. With the colormap, the median values and concentrations for the distinct collected parameters are plotted over the road network. Utilizing GIS road network datasets, the collected geo-localized datasets were mapped over the shapefiles binning based on proximity. In the plots shown above, a binning of $\sim 100 \mathrm{~m}$ was performed. That is, the median of the data points contained within a $\sim 50 \mathrm{~m}$ radius circle were computed. Overall, as shown in the displayed data, the highest concentrations were observed in the main intersections in the city and in the main traffic arteries that connect the campus to US Route 1. The presence of the lake and its influence on specific humidity and temperature were also consistent.
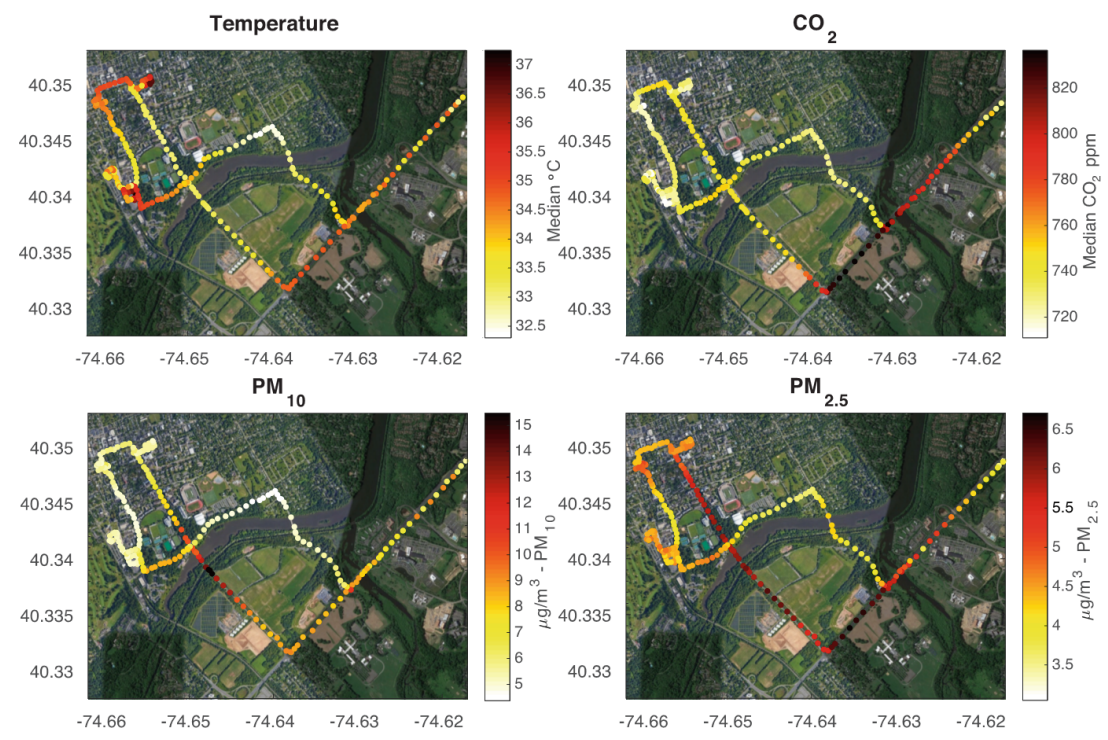

Fig. 6. Averaged Temperature, $\mathrm{CO}_{2}, \mathrm{PM}_{2.5}$ and $\mathrm{PM}_{10}$ data for the afternoon of July $17^{\text {th }} 2019$.

In Fig. 7, a 3-dimensional visualization of the $\mathrm{CO}_{2}$ data collected in one of the measuring trips is displayed. The purpose of this visualization is to enable an intuitive understanding of the correlation between building density and concentration levels as well as the distinct road characteristics within the trajectories. The model comprises the whole extent of the campus until US Route 1. 


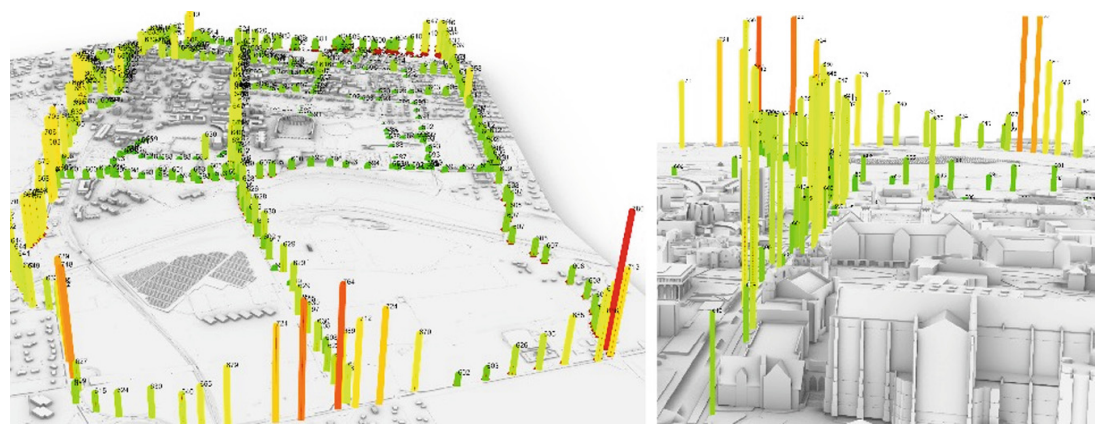

Fig. 7. $\mathrm{CO}_{2}$ concentration visualization within the Princeton Uiversity Campus.

As shown in Fig. 8 the collected data were also made available in real time in the Princeton University website must.princeton.edu. Utilizing the google maps API, the environmental data are displayed together with the google traffic information, and map or satellite view. In the web app, a scatter plot displaying the data points collected by the sensing kits or an interpolated plot using a kriging interpolation is enabled. As shown in Fig. 8, a mobile app was also developed to display the environmental quality information as an interpolated gradient, with specific information on every road intersection.
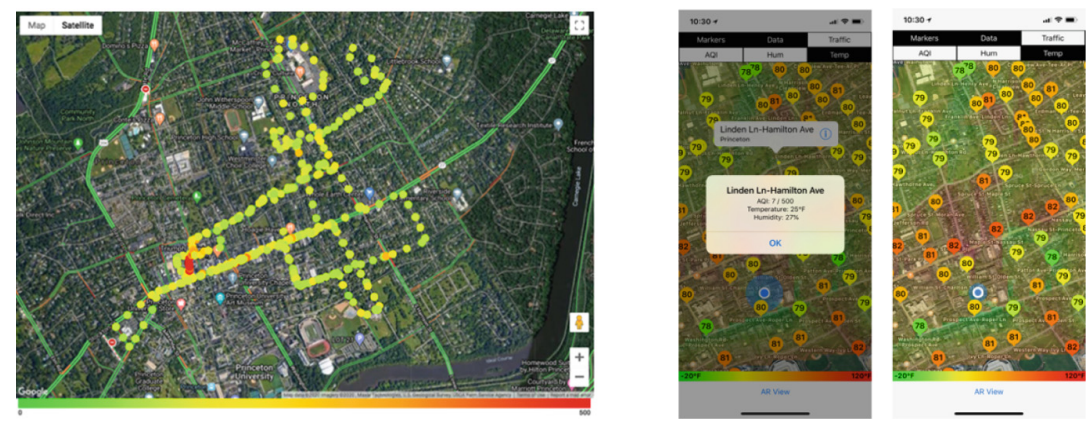

Fig. 8. The plot to the left displays the web application showing the scatter plot of an hour of measuring trip trajectory for the air quality parameter PM2.5. The plots to the right show the mobile application showing the environmental quality within Princeton University Campus (Built with google maps API). 


\subsection{Summary}

An autonomous and affordable Mobile Urban Sensing Technology (MUST) to capture the urban spatial gradients at the neighborhood scale has been presented. The challenges to obtain accurate high spatial resolution measurements have been discussed, and the design criteria followed to enable a fast-response sensing kit mechanical design that overcomes these challenges have been described. Strategies to enhance enclosure resilience against heavy rainfall and overheating have also been discussed. The results obtained in an experimental campaign developed within Princeton University campus during July 2019 have been presented, along with environmental data visualization strategies to communicate the data to wider audiences. The study has shown that with a small investment and maintenance control, urban planners, policy makers and municipalities could make use of high spatial resolution urban environmental data to aim for environmental justice in the most compromised neighborhoods in our cities. Given the recent advances in computer vision technologies, it is becoming increasingly more plausible to couple affordable visible and IR cameras and environmental sensing kits to train a computer vision algorithm to emulate air quality information, or to develop a real-time data quality control mechanism, citizen exposure diagnosis, or pollution source detection method. Such technologies are being incorporated in the new generation of MUST sensing kits.

Acknowledgements. Authors would like to acknowledge the support of the Princeton University Campus as Lab grant, and the Army Research Office under award \# W911NF2010216 (Program Manager Julia Barzyk). We would also like to acknowledge the contribution of Traci Mathieu who developed the mobile app.

\section{References}

1. Apte, J.S., Messier, K.P., Gani, S., Brauer, M., Kirchstetter, T.W., Lunden, M.M., Marshall, J.D., Portier, C., Vermeulen, R.C., Hamburg, S.P.: High-resolution air pollution mapping with Google street view cars: exploiting big data. Environ. Sci. Technol. 51, 6999-7008 (2017)

2. Llaguno-Munitxa, M., Bou-Zeid, E.: Environmental Neighborhoods and Their Spatial Extent. IOP Publishing, Environmental Rersearch Letters (2020)

3. Brugge, D., Durant, J.L., Rioux, C.: Near-highway pollutants in motor vehicle exhaust: a review of epidemiologic evidence of cardiac and pulmonary health risks. Environ Health $\mathbf{6}$, 23 (2007)

4. Cramer, J., Jorgensen, J.T., Hoffmann, B., Loft, S., Brauner, E.V., Prescott, E., Ketzel, M., Hertel, O., Brandt, J., Jensen, S.S., Backalarz, C., Simonsen, M.K., Andersen, Z.J.: LongTerm exposure to air pollution and incidence of myocardial infarction: a Danish nurse cohort study. Environ. Health Perspect. 128, 5 (2020)

5. Environmental Defense Fund: Why new sensor tech is critical for tackling air pollution around the globe (2020). https://www.edf.org/airqualitymaps

6. C40 Cities: Air Quality Initiative (2018). https://www.c40.org/networks/air-quality

7. Chen, L.J., Hsu, W., Cheng, M., Lee, H.C.: DEMO: LASS: a location-aware sensing system for participatory PM2.5 monitoring MobiSys' Companion. In: Proceedings of the 14th Annual International. Conference on Mobile Systems, Applications, and Services Companion, pp. 98 98 (2016) 
8. Environmental Defense Fund: Clean Air Innovation (2019). https://business.edf.org/insights/ future-fleets-collecting-air-pollution-data/

9. Schmidt, W.: Die Vertilung der Minimurntemperaturen in derl Frostnacht des 12. 5.19.27 im Gemeindegebiet von Wien. In Fortschritte der Landwirtschaft (1927)

10. Peppler, A.: Die Temperaturverhaltnise von Karlsruhe an heissen Sommertagen. Deutsche Meteorologisches (1929)

11. Budel, H., Wolf, G.: Sonderklima der stadte geog. Wochenschr 1, 25-31 (1933)

12. Van Poppel, M., Peters, J., Bleux, N.: Methodology for setup and data processing of mobile air quality measurements to assess the spatial variability of concentrations in urban environments. Environ. Pollut. 183, 224-233 (2013)

13. Levy, I., Mihele, C., Lu, G., Narayan, J., Brook, J.R.: Evaluating multipollutant exposure and urban air quality: pollutant interrelationships, neighborhood variability, and nitrogen dioxide as a proxy pollutant. Environ. Health Perspect. 122, 65-72 (2014)

14. BBC News: London air pollution: google Street View cars to carry monitors. (2019). https:// www.bbc.com/news/uk-england-london-46878067

15. Messier, K.P., et al.: Mapping air pollution with google street view cars: efficient approaches with mobile monitoring and land use regression. Environ. Sci. Technol. 52, 12563-12572 (2018)

16. Miller, D.J., Actkinson, B., Padilla, L., Griffin, R.J., Moore, K., Lewis, P.G.T., GardnerFrolick, R., Craft, E., Portier, C.J., Hamburg, S.P., Alvarez, R.A.: Characterizing elevated urban air pollutant patterns with mobile monitoring in Houston, Texas. Environ. Sci. Technol. 54-4, 2133-2142 (2020)

17. Llaguno Munitxa, M., Bou-Zeid, E., Bogosian, B., Al Tair, A., Radcliff, D., Fisher, S., Ryu, Y.: Sensing and information technologies for the environment (SITE); hardware and software innovations in mobile sensing applications. In: International Building Physics Conference 2018, Syracuse, NY, USA (2018)

18. Llaguno-Munitxa, M., Bogosian, M., Bou-Zeid, E., Fisher, S., Al-Tair, A., Radcliff, D.: Seoul On-Air: Augmented Environments for Urban Activism, Imminent Commons: The Expanded City. Seoul Biennale of Architecture and Urbanism 2017 (2017)

19. Jimenez, J.M.: High Reynolds Number Flows about Bodies of Revolution with Application to Submarines and Torpedoes. PhD, Deparment of Engineering, Princeton University (2007)

Open Access This chapter is licensed under the terms of the Creative Commons Attribution 4.0 International License (http://creativecommons.org/licenses/by/4.0/), which permits use, sharing, adaptation, distribution and reproduction in any medium or format, as long as you give appropriate credit to the original author(s) and the source, provide a link to the Creative Commons license and indicate if changes were made.

The images or other third party material in this chapter are included in the chapter's Creative Commons license, unless indicated otherwise in a credit line to the material. If material is not included in the chapter's Creative Commons license and your intended use is not permitted by statutory regulation or exceeds the permitted use, you will need to obtain permission directly from the copyright holder.

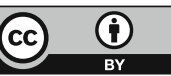

